\title{
A New Hybrid Distance-Based Similarity Measure for Refined Neutrosophic Sets and its Application in Medical Diagnosis
}

\author{
${ }^{1}$ VakkasUluçay, ${ }^{2}$ Adil Kılıç, ${ }^{3}$ Memet Şahin and ${ }^{4}$ Harun Deniz \\ ${ }^{1}$ Köklüce Neighbourhood, Araban, Gaziantep27310-Turkey \\ ${ }^{2,3,4}$ Department of Mathematics, Gaziantep University, Gaziantep27310-Turkey \\ ${ }^{*}$ Corresponding author:vulucay27@gmail.com
}

Article history

Received: 6 March 2018

Received in revised form: 7 August 2018

Accepted: 12 November 2018

Published on line: 1 April 2019

\begin{abstract}
The aim of this paper is to introduce a new distance-based similarity measure for refined neutrosophic sets. The properties of the proposed new distance-based similarity measure have been studied and the findings are applied in medical diagnosis of some diseases with a common set of symptoms.
\end{abstract}

Keywords Neutrosophic sets; refined neutrosophic sets; similarity measure decision making; medical diagnosis.

Mathematics Subject Classification $03 \mathrm{~B} 52$

\section{Introduction}

The vagueness or uncertainty representation of imperfect knowledge becomes a crucial issue in the areas of computer science and artificial intelligence. To deal with the uncertainty, the fuzzy set proposed by Zadeh [1] allows the uncertainty of a set with a membership degree between 0 and 1. Then, Atanassov [2] introduced an intuitionistic Fuzzy set (IFS) as a generalization of the Fuzzy set. The IFS represents the uncertainty with respect to both membership and nonmembership. However, it can only handle incomplete information but not the indeterminate and inconsistent information which exists commonly in real situations. Therefore, Smarandache [3] proposed a neutrosophic set. It can independently express truth-membership degree, indeterminacy-membership degree, and false membership degree and deal with incomplete, indeterminate, and inconsistent information. Also, several generalization of the set theories made such as fuzzy multi-set theory [4, 5], intuitionsitic fuzzy multi-set theory [6-10] and refined neutrosophic set theory [11-20]. Many research treating imprecision and uncertainty have been developed and studied. Since then, it is applied to various areas, such as decision making problems [21-44].

Another generalization of above theories that is relevant for our work is single valued neutrosophic refined (multi) set theory by introduced Ye [45] which contain a few different values. A single valued neutrosophic multi set theory have truth-membership sequence 
$\left(\mu_{\mathcal{A}}^{1}(u), \mu_{\mathcal{A}}^{2}(u), \ldots \mu_{\mathcal{A}}^{p}(u)\right)$, indeterminacy-membership sequence $\left(v_{\mathcal{A}}^{1}(u), v_{\mathcal{A}}^{2}(u), \ldots v_{\mathcal{A}}^{p}(u)\right)$ and falsity-membership sequence $\left(w_{\mathcal{A}}^{1}(u), w_{\mathcal{A}}^{2}(u), \ldots w_{\mathcal{A}}^{p}(u)\right)$ of the element $u \in \mathcal{U}$

The paper is organized as follows: In section 2 , introduces some concepts and basic operations are reviewed. In section 3, presents a new distance-based similarity measure for refined neutrosophic sets and investigates their properties. In section 4 , the similarity measures are applied to medicine diagnosis. Finally, Conclusions and further research are contained

\section{Preliminaries}

Definition 1 [2] Let $\mathcal{U}$ be a universe. $A$ neutrosophic sets $A$ over $\mathcal{U}$ is defined by

$$
\mathcal{A}=\left\{\prec,\left(\mu_{\mathcal{A}}(\mu), v_{\mathcal{A}}(\mu), w_{\mathcal{A}} \succ: u \in \mathcal{U}\right\}\right.
$$

where, $\mu_{\mathcal{A}}(u), v_{\mathcal{A}}(u)$ and $w_{\mathcal{A}}(u)$ are called truth-membership function, indeterminacy-membership function and falsity- membership function, respectively. They are respectively defined by

$$
\left.\mu_{\mathcal{A}}: \mathcal{U} \rightarrow\right]^{-} 0,1^{+}\left[, v_{\mathcal{A}}: \mathcal{U} \rightarrow\right]^{-} 0,1^{+}\left[, \quad w_{\mathcal{A}}: \mathcal{U} \rightarrow\right]^{-} 0,1^{+}[
$$

such that $0^{-} \leq \mu_{\mathcal{A}}(u)+v_{\mathcal{A}}(u)+w_{\mathcal{A}}(u) \leq 3^{+}$

Definition 2 [19] Let $\mathcal{U}$ be a universe. A single valued neutrosophic set (SVN-set) over $\mathcal{U}$ is a neutrosophic set over $\mathcal{U}$, but the truth-membership function, indeterminacy-membership function and falsity- membership function are respectively defined by

$$
\mu_{\mathcal{A}}: \mathcal{U} \rightarrow[0,1], v_{A}: U \rightarrow[0,1], \quad w_{\mathcal{A}}: \mathcal{U} \rightarrow[0,1]
$$

Such that $0 \leq \mu_{\mathcal{A}}(u)+v_{\mathcal{A}}(u)+w_{\mathcal{A}}(u) \leq 3$

Definition 3 [32] Let $\mathcal{U}$ be a universe. A neutrosophic multiset set (Nms) $A$ on $U$ can be defined as follows:

$$
\begin{aligned}
& \mathcal{A}=\{ \\
& \prec u\left(\mu_{\mathcal{A}}^{1}(u), \mu_{\mathcal{A}}^{2}(u), \ldots \mu_{A}^{p}(u)\right)\left(v_{\mathcal{A}}^{1}(u), v_{\mathcal{A}}^{2}(u), \ldots v_{\mathcal{A}}^{p}(u)\right)\left(w_{\mathcal{A}}^{1}(u), w_{\mathcal{A}}^{2}(u), \ldots w_{\mathcal{A}}^{p}(u)\right) \\
& \succ: u \in \mathcal{U}\}
\end{aligned}
$$

where,

$$
\begin{aligned}
\mu_{\mathcal{A}}^{1}(u) \mu_{\mathcal{A}}^{2}(u), \ldots \mu_{\mathcal{A}}^{p}(u): \mathcal{U} & \rightarrow[0,1] \\
v_{\mathcal{A}}^{1}(u) v_{\mathcal{A}}^{2}(u), \ldots v_{\mathcal{A}}^{p}(u): \mathcal{U} & \rightarrow[0,1]
\end{aligned}
$$

and

$$
w_{\mathcal{A}}^{1}(u) w_{\mathcal{A}}^{2}(u), \ldots w_{\mathcal{A}}^{p}(u): \mathcal{U} \rightarrow[0,1]
$$

such that

$$
0 \leq \sup \mu_{\mathcal{A}}^{i}(u)+\sup v_{\mathcal{A}}^{i}(u)+\sup w_{\mathcal{A}}^{i}(u) \leq 3
$$

$(i=1,2, \ldots, P)$ and

$$
\left(\mu_{\mathcal{A}}^{1}(u), \mu_{\mathcal{A}}^{2}(u), \ldots, \mu_{\mathcal{A}}^{p}(u)\right)\left(v_{\mathcal{A}}^{1}(u), v_{\mathcal{A}}^{2}(u), \ldots, v_{\mathcal{A}}^{p}(u)\right) \text { and }\left(w_{\mathcal{A}}^{1}(u), w_{\mathcal{A}}^{2}(u), \ldots, w_{\mathcal{A}}^{p}(u)\right)
$$


is the truth-membership sequence, indeterminacy-membership sequence and falsity- membership sequence of the element $u$ respectively. Also, $\mathrm{P}$ is called the dimension (cardinality) of $\operatorname{Nms} \mathcal{A}$ denoted $d(\mathcal{A})$ We arrange the truth-membership sequence in decreasing order but the corresponding indeterminacy-membership and falsity-membership sequence may not be in decreasing or increasing order.

The set of all Neutrosophic multisets on $\mathcal{U}$ is denoted by $\operatorname{NMS}(\mathcal{U})$.

Definition $4[11,19,38]$ Let $\mathcal{A}, \mathcal{B} \in N M S(\mathcal{U})$. Then,

(1) $\mathcal{A}$ is said to be Nm-subset of $\mathcal{B}$ is denoted by $\mathcal{A} \widetilde{\subseteq} \mathcal{B}$ if $\mu_{\mathcal{A}}^{i}(u) \leq \mu_{\mathcal{B}}^{i}(u)$ $v_{\mathcal{A}}^{i}(u) \geq v_{\mathcal{B}}^{i}(u), \quad w_{\mathcal{A}}^{i}(u) \geq w_{\mathcal{B}}^{i}(u), \quad \forall u \in \mathcal{U}$ and $i=1,2, \ldots P$

(2) $\mathcal{A}$ is said to be neutrosophic equal of $\mathcal{B}$ is denoted by $\mathcal{A}=\mathcal{B}$ if $\mu_{\mathcal{A}}^{i}(u)=\mu_{\mathcal{B}}^{i}(u)$ $v_{\mathcal{A}}^{i}(u)=v_{\mathcal{B}}^{i}(u) w_{\mathcal{A}}^{i}(u)=w_{\mathcal{B}}^{i}(u), \quad \forall u \in \mathcal{U}$ and $i=1,2, \ldots P$

(3) The complement of $\mathcal{A}$ denoted by $\mathcal{A}^{\tilde{c}}$ and is defined by

$$
\begin{aligned}
& A^{\tilde{c}}= \\
& \prec u\left(w_{A}^{1}(u), w_{A}^{2}(u), \ldots, w_{A}^{p}(u)\right)\left(v_{A}^{1}(u), v_{A}^{2}(u), \ldots v_{A}^{p}(u)\right)\left(\mu_{A}^{1}(u), \mu_{A}^{2}(u), \ldots \mu_{A}^{p}(u)\right) \\
& \succ: u \in \mathcal{U}
\end{aligned}
$$

(4) If $\mu_{\mathcal{A}}^{i}(u)=0$ and $v_{\mathcal{A}}^{i}(u)=w_{\mathcal{A}}^{i}(u)=1$ for all $u \in \mathcal{U}$ and $i=1,2, \ldots P$ then $\mathcal{A}$ is called null ns-set and denoted by $\Phi$

(5) If $\mu_{\mathcal{A}}^{i}(u)=1$ and $v_{\mathcal{A}}^{i}(u)=w_{\mathcal{A}}^{i}(u)=0$ for all $u \in \mathcal{U}$ and $i=1,2, \ldots P$ then $A$ is called universal ns-set and denoted by $\widetilde{\mathcal{U}}$

(6) The union of $\mathcal{A}$ and $\mathcal{B}$ is denoted by $\mathcal{A} \widetilde{\cup}=\mathcal{C}$ and is defined by

$$
\begin{aligned}
& \mathcal{C}=\{ \\
& \prec u\left(\mu_{\mathcal{C}}^{1}(u), \mu_{\mathcal{C}}^{2}(u), \ldots \mu_{\mathcal{C}}^{p}(u)\right)\left(v_{\mathcal{C}}^{1}(u), v_{\mathcal{C}}^{2}(u), \ldots v_{\mathcal{C}}^{p}(u)\right)\left(w_{\mathcal{C}}^{1}(u), w_{\mathcal{C}}^{2}(u), \ldots w_{\mathcal{C}}^{p}(u)\right) \succ: u \\
& \in \mathcal{U}\}
\end{aligned}
$$

Where $\mu_{\mathcal{C}}^{i}=\mu_{\mathcal{A}}^{i}(u) \vee \mu_{\mathcal{B}}^{i}(u), v_{\mathcal{C}}^{i}=v_{\mathcal{A}}^{i}(u) \wedge v_{\mathcal{B}}^{i}(u), w_{\mathcal{C}}^{i}=w_{\mathcal{A}}^{i}(u) \wedge w_{\mathcal{B}}^{i}(u)$ $\forall u \in \mathcal{U}$ and $i=1,2, \ldots P$

(7) The intersection of $\mathcal{A}$ and $\mathcal{B}$ is denoted by $\mathcal{A} \widetilde{\cap} \mathcal{B}=\mathcal{D}$ and is defined by

$$
\begin{aligned}
& \mathcal{D}=\{ \\
& \prec u\left(\mu_{\mathcal{D}}^{1}(u), \mu_{\mathcal{D}}^{2}(u), \ldots \mu_{\mathcal{D}}^{p}(u)\right)\left(v_{\mathcal{D}}^{1}(u), v_{\mathcal{D}}^{2}(u), \ldots v_{D}^{p}(u)\right)\left(w_{\mathcal{D}}^{1}(u), w_{\mathcal{D}}^{2}(u), \ldots w_{\mathcal{D}}^{p}(u)\right) \\
& \succ: u \in \mathcal{U}\}
\end{aligned}
$$

where $\mu_{\mathcal{D}}^{i}=\mu_{\mathcal{A}}^{i}(u) \vee \mu_{\mathcal{B}}^{i}(u), v_{\mathcal{D}}^{i}=v_{\mathcal{A}}^{i}(u) \wedge v_{\mathcal{B}}^{i}(u), w_{\mathcal{D}}^{i}=w_{\mathcal{A}}^{i}(u) \wedge w_{\mathcal{B}}^{i}(u), \forall u \in \mathcal{U}$ and $i=1,2, \ldots P$

(8) The addition of $\mathcal{A}$ and $\mathcal{B}$ is denoted by $\mathcal{A} \widetilde{+B}=\mathcal{U}_{1}$ and is defined by

$$
\begin{aligned}
& \mathcal{U}_{1}=\{ \\
& \prec u\left(\mu_{\mathcal{U}_{1}}^{1}(u), \mu_{\mathcal{U}_{1}}^{2}(u), \ldots \mu_{\mathcal{U}_{1}}^{p}(u)\right)\left(v_{\mathcal{U}_{1}}^{1}(u), v_{\mathcal{U}_{1}}^{2}(u), \ldots v_{\mathcal{U}_{1}}^{p}(u)\right)\left(w_{\mathcal{U}_{1}}^{1}(u), w_{\mathcal{U}_{1}}^{2}(u), \ldots w_{\mathcal{U}_{1}}^{p}(u)\right) \\
& \succ: u \in \mathcal{U}
\end{aligned}
$$

where $\mu_{\mathcal{U}_{1}}^{i}=\mu_{\mathcal{A}}^{i}(u)+\mu_{\mathcal{B}}^{i}(u)-\mu_{\mathcal{A}}^{i}(u) \mu_{\mathcal{B}}^{i}(u) v_{U_{1}}^{i}=v_{\mathcal{A}}^{i}(u) v_{\mathcal{B}}^{i}(u), w_{\mathcal{U}_{1}}^{i}=w_{\mathcal{A}}^{i}(u) w_{\mathcal{B}}^{i}(u) \forall u \in \mathcal{U}$ and $i=1,2, \ldots P$ 
(9) The multiplication of $A$ and $B$ is denoted by $\mathcal{A} \widetilde{\mathcal{X}} \mathcal{B}=\mathcal{U}_{2}$ and is defined by

$$
\begin{aligned}
& \mathcal{U}_{2}=\{ \\
& \prec u\left(\mu_{\mathcal{U}_{2}}^{1}(u), \mu_{\mathcal{U}_{2}}^{2}(u), \ldots \mu_{\mathcal{U}_{2}}^{p}(u)\right)\left(v_{\mathcal{U}_{2}}^{1}(u), v_{\mathcal{U}_{2}}^{2}(u), \ldots v_{\mathcal{U}_{2}}^{p}(u)\right)\left(w_{\mathcal{U}_{2}}^{1}(u), w_{\mathcal{U}_{2}}^{2}(u), \ldots w_{\mathcal{U}_{2}}^{p}(u)\right) \\
& \succ: u \in \mathcal{U} \\
& \text { where } \mu_{\mathcal{U}_{2}}^{i}=\mu_{\mathcal{A}}^{i}(u) \mu_{\mathcal{B}}^{i}(u), v_{\mathcal{U}_{2}}^{i}=v_{\mathcal{A}}^{i}(u)+v_{\mathcal{B}}^{i}(u)-v_{\mathcal{A}}^{i}(u) v_{\mathcal{B}}^{i}(u) w_{\mathcal{U}_{2}}^{i}=w_{\mathcal{A}}^{i}(u)+w_{\mathcal{B}}^{i}(u)- \\
& w_{\mathcal{A}}^{i}(u) w_{\mathcal{B}}^{i}(u) \forall u \in \mathcal{U} \text { and } i=1,2, \ldots P \\
& \text { Here } \vee \wedge, \wedge,+,,- \text { denotes maximum, minimum, addition, multiplication, subtraction of } \\
& \text { real numbers respectively. } \\
\text { Definition } 5[12] \text { Let } & \\
& \mathcal{A}=\{ \\
& \prec u\left(\mu_{\mathcal{A}}^{1}(u), \mu_{\mathcal{A}}^{2}(u), \ldots \mu_{\mathcal{A}}^{p}(u)\right)\left(v_{\mathcal{A}}^{1}(u), v_{\mathcal{A}}^{2}(u), \ldots v_{\mathcal{A}}^{p}(u)\right)\left(w_{\mathcal{A}}^{1}(u), w_{\mathcal{A}}^{2}(u), \ldots w_{\mathcal{A}}^{p}(u)\right) \\
& \succ: u \in \mathcal{U}
\end{aligned}
$$

and

$$
\begin{aligned}
& \mathcal{B}=\{ \\
& \prec u\left(\mu_{\mathcal{B}}^{1}(u), \mu_{\mathcal{B}}^{2}(u), \ldots \mu_{\mathcal{B}}^{p}(u)\right)\left(v_{\mathcal{B}}^{1}(u), v_{\mathcal{B}}^{2}(u), \ldots v_{\mathcal{B}}^{p}(u)\right)\left(w_{\mathcal{A}}^{1}(u), w_{\mathcal{A}}^{2}(u), \ldots w_{\mathcal{A}}^{p}(u)\right) \\
& \succ: u \in \mathcal{U}
\end{aligned}
$$

And be two NMSs, then the normalized hamming distance between $\mathcal{A}$ and $\mathcal{B}$ can be defined as follows:

$$
d_{p}(\mathcal{A}, \mathcal{B})=\left\{\frac{1}{3} \sum_{i=1}^{p} \omega_{i}\left[\left|\mu_{\mathcal{A}}\left(u_{i}\right)-\mu_{\mathcal{B}}\left(u_{i}\right)\right|^{p}+\left|v_{\mathcal{A}}\left(u_{i}\right)-v_{\mathcal{B}}\left(u_{i}\right)\right|^{p}+\left|w_{\mathcal{A}}\left(u_{i}\right)-w_{\mathcal{B}}\left(u_{i}\right)\right|^{p}\right]\right\}^{1 / p}
$$

where $\mathcal{A}, \mathcal{B}$ are two SVNSs $p>0, w_{i}(i=1,2, \ldots, p)$ are the weight of the element $x_{i}(i=1,2, \ldots, p)$ with $w_{i} \geq 0$ and $\sum_{i=1}^{n} w_{i}=1$.

In the next section, we will define a new Hybrid Distance-Based Similarity Measures for Refined Neutrosophic Sets (RNSs)

\section{Hybrid Distance-Based Similarity Measures for Refined Neutrosophic Sets}

Definition 6 For two refined neutrosophic sets $\mathcal{A}$ and $\mathcal{B}$ in a universe of discourse which are denoted by

$$
\begin{aligned}
& \mathcal{A}=\{ \\
& \prec u\left(\mu_{\mathcal{A}}^{1}(u), \mu_{\mathcal{A}}^{2}(u), \ldots \mu_{\mathcal{A}}^{p}(u)\right)\left(v_{\mathcal{A}}^{1}(u), v_{\mathcal{A}}^{2}(u), \ldots v_{\mathcal{A}}^{p}(u)\right)\left(w_{\mathcal{A}}^{1}(u), w_{\mathcal{A}}^{2}(u), \ldots w_{\mathcal{A}}^{p}(u)\right) \\
& \succ: u \in \mathcal{U}
\end{aligned}
$$


and

$$
\begin{aligned}
& \mathcal{B}=\{ \\
& \prec u\left(\mu_{\mathcal{B}}^{1}(u), \mu_{\mathcal{B}}^{2}(u), \ldots \mu_{\mathcal{B}}^{p}(u)\right)\left(v_{\mathcal{B}}^{1}(u), v_{\mathcal{B}}^{2}(u), \ldots v_{\mathcal{B}}^{p}(u)\right)\left(w_{\mathcal{B}}^{1}(u), w_{\mathcal{B}}^{2}(u), \ldots w_{\mathcal{B}}^{p}(u)\right) \\
& \succ: u \in \mathcal{U}
\end{aligned}
$$

$\mu_{\mathcal{A}}^{i}(u) \mu_{\mathcal{B}}^{i}(u) v_{\mathcal{A}}^{i}(?) v_{\mathcal{B}}^{i}(u) w_{\mathcal{A}}^{i}(u) w_{\mathcal{B}}^{i}(u) \in[0,1]$ for every $i=1,2, \ldots P$. Let us consider the weight $\omega_{i} i=1,2, \ldots P$ with $\omega_{i} \geq 0, i=1,2, \ldots P$ and $\sum_{i=1}^{P} \omega_{i}=1$.

Then, we define the refined generalized neutrosophic weighted distance measure:

$$
d_{P}(\mathcal{A}, \mathcal{B})=\left\{\frac{1}{3} \sum_{i=1}^{P} \omega_{i}\left[\left|\mu_{\mathcal{A}}^{i}\left(u_{i}\right)-\mu_{\mathcal{B}}^{i}\left(u_{i}\right)\right|^{P}+\left|v_{\mathcal{A}}^{i}\left(u_{i}\right)-v_{\mathcal{B}}^{i}\left(u_{i}\right)\right|^{P}+\left|w_{\mathcal{A}}^{i}\left(u_{i}\right)-w_{\mathcal{B}}^{i}\left(u_{i}\right)\right|^{P}\right]\right\}^{1 / p}
$$

where $P>0$.

As the Hamming distance and Euclidean distance, which are two typical distance measures, are usually used in practical applications when $P=1,2$ we can obtain the refined neutrosophic weighted Hamming distance and the refined neutrosophic weighted Euclidean distance, respectively, as follows:

$$
\begin{aligned}
& d(\mathcal{A}, \mathcal{B})=\frac{1}{3} \sum_{i=1}^{P} \omega_{i}\left[\left|\mu_{\mathcal{A}}^{i}\left(u_{i}\right)-\mu_{\mathcal{B}}^{i}\left(u_{i}\right)\right|+\left|v_{\mathcal{A}}^{i}\left(u_{i}\right)-v_{\mathcal{B}}^{i}\left(u_{i}\right)\right|+\left|w_{\mathcal{A}}^{i}\left(u_{i}\right)-w_{\mathcal{B}}^{i}\left(u_{i}\right)\right|,\right. \\
& d_{2}(\mathcal{A}, \mathcal{B})=\left\{\frac{1}{3} \sum_{i=1}^{P} \omega_{i}\left[\left|\mu_{\mathcal{A}}^{i}\left(u_{i}\right)-\mu_{\mathcal{B}}^{i}\left(u_{i}\right)\right|^{2}+\left|v_{\mathcal{A}}^{i}\left(u_{i}\right)-v_{B}^{i}\left(u_{i}\right)\right|^{2}+\left|w_{\mathcal{A}}^{i}\left(u_{i}\right)-w_{\mathcal{B}}^{i}\left(u_{i}\right)\right|^{2}\right]\right\}^{1 / p} .
\end{aligned}
$$

Therefore, equations (2) and (3) are the special cases of equation (1). Then, for the distance measure, we have the following proposition.

Proposition 7 The distance measured $_{p}(A, B)$ for $p>0$ satisfies the following properties:

$(\mathrm{H} 1) \quad 0 \leq d_{p}(\mathcal{A}, \mathcal{B}) \leq 1$

(H2) $d_{p}(\mathcal{A}, \mathcal{B})=0$ if and only if $\mathcal{A}=\mathcal{B}$

(H3) $d_{p}(\mathcal{A}, \mathcal{B})=d_{p}(\mathcal{B}, \mathcal{A})$;

(H4) If $\subseteq \mathcal{B} \subseteq \mathcal{C}, \mathcal{A}_{3}$ is are fined neutrosophicin $\mathcal{U}$, then $d_{p}(\mathcal{A}, \mathcal{B}) \leq d_{p}(A C)$ andd $d_{p}(\mathcal{B}, \mathcal{C}) \leq$ $d_{p}(\mathcal{A}, \mathcal{C})$

Proof: It is easy to see that $d_{p}(\mathcal{A}, \mathcal{B})$ satisfies the properties $(H 1)-(H 3)$ Therefore, we only prove (H4). Let $\mathcal{A} \subseteq \mathcal{B} \subseteq \mathcal{C}$, then

$$
\mu_{\mathcal{A}}^{i}(u) \leq \mu_{\mathcal{B}}^{i}(u) \leq \mu_{\mathcal{C}}^{i}(u), v_{\mathcal{A}}^{i}(u) \geq v_{\mathcal{B}}^{i}(u) \geq \mu_{\mathcal{C}}^{i}(u), w_{\mathcal{A}}^{i}(u) \geq w_{\mathcal{B}}^{i}(u) \geq \mu_{\mathcal{C}}^{i}(u), \quad \forall u_{i} \in \mathcal{U}
$$

and $i=1,2, \ldots P$ we obtain following relations:

$$
\begin{gathered}
\left|\mu_{\mathcal{A}}^{i}\left(u_{i}\right)-\mu_{\mathcal{B}}^{i}\left(u_{i}\right)\right|^{P} \leq\left|\mu_{\mathcal{A}}^{i}\left(u_{i}\right)-\mu_{\mathcal{C}}^{i}\left(u_{i}\right)\right|^{P} ;\left|\mu_{\mathcal{B}}^{i}\left(u_{i}\right)-\mu_{\mathcal{C}}^{i}\left(u_{i}\right)\right|^{P} \leq\left|\mu_{\mathcal{A}}^{i}\left(u_{i}\right)-\mu_{\mathcal{C}}^{i}\left(u_{i}\right)\right|^{P} \\
\left|v_{\mathcal{A}}^{i}\left(u_{i}\right)-v_{\mathcal{B}}^{i}\left(u_{i}\right)\right|^{P} \leq\left|v_{\mathcal{A}}^{i}\left(u_{i}\right)-v_{\mathcal{C}}^{i}\left(u_{i}\right)\right|^{P} ;\left|v_{\mathcal{B}}^{i}\left(u_{i}\right)-v_{\mathcal{C}}^{i}\left(u_{i}\right)\right|^{P} \leq\left|v_{\mathcal{A}}^{i}\left(u_{i}\right)-v_{\mathcal{C}}^{i}\left(u_{i}\right)\right|^{P} \\
\left|w_{\mathcal{A}}^{i}\left(u_{i}\right)-w_{\mathcal{B}}^{i}\left(u_{i}\right)\right|^{P} \leq\left|w_{\mathcal{A}}^{i}\left(u_{i}\right)-w_{\mathcal{C}}^{i}\left(u_{i}\right)\right|^{P} ;\left|w_{B}^{i}\left(u_{i}\right)-w_{\mathcal{C}}^{i}\left(u_{i}\right)\right|^{P} \leq\left|w_{\mathcal{A}}^{i}\left(u_{i}\right)-w_{\mathcal{C}}^{i}\left(u_{i}\right)\right|^{P}
\end{gathered}
$$


Hence,

$$
\begin{aligned}
& \quad\left|\mu_{\mathcal{A}}^{i}\left(u_{i}\right)-\mu_{\mathcal{B}}^{i}\left(u_{i}\right)\right|^{P}+\left|v_{\mathcal{A}}^{i}\left(u_{i}\right)-v_{\mathcal{B}}^{i}\left(u_{i}\right)\right|^{P}+\left|w_{\mathcal{A}}^{i}\left(u_{i}\right)-w_{\mathcal{B}}^{i}\left(u_{i}\right)\right|^{P} \\
& \leq\left|\mu_{\mathcal{A}}^{i}\left(u_{i}\right)-\mu_{\mathcal{C}}^{i}\left(u_{i}\right)\right|^{P}+\left|v_{\mathcal{A}}^{i}\left(u_{i}\right)-v_{\mathcal{C}}^{i}\left(u_{i}\right)\right|^{P}+\left|w_{\mathcal{A}}^{i}\left(u_{i}\right)-w_{\mathcal{C}}^{i}\left(u_{i}\right)\right|^{P} \\
& \left|\mu_{\mathcal{B}}^{i}\left(u_{i}\right)-\mu_{\mathcal{C}}^{i}\left(u_{i}\right)\right|^{P}+\left|v_{\mathcal{B}}^{i}\left(u_{i}\right)-v_{\mathcal{C}}^{i}\left(u_{i}\right)\right|^{P}+\left|w_{\mathcal{B}}^{i}\left(u_{i}\right)-w_{\mathcal{C}}^{i}\left(u_{i}\right)\right|^{P} \\
& \leq\left|\mu_{\mathcal{A}}^{i}\left(u_{i}\right)-\mu_{\mathcal{C}}^{i}\left(u_{i}\right)\right|^{P}+\left|v_{\mathcal{A}}^{i}\left(u_{i}\right)-v_{\mathcal{C}}^{i}\left(u_{i}\right)\right|^{P}+\left|w_{\mathcal{A}}^{i}\left(u_{i}\right)-w_{\mathcal{C}}^{i}\left(u_{i}\right)\right|^{P}
\end{aligned}
$$

$d_{\lambda}(\mathcal{A}, \mathcal{C}) \geq d_{\lambda}(\mathcal{A}, \mathcal{B})$ and $d_{\lambda}(\mathcal{A}, \mathcal{C}) \geq d_{\lambda}(\mathcal{B}, \mathcal{C})$ for $\lambda>0$.

Example 8: Assume that we have the following three refined neutrosophic weighted Hamming distance and the refined neutrosophic weighted Euclidean distance, in a universe of discourse $u \in \mathcal{U}$ : let $\omega_{1}=0.2, \omega_{2}=0.4, \omega_{3}=0.4$.

$$
\begin{aligned}
& \mathcal{A}=\langle u,(0.8,0.5,0.6),(0.3,0.1,0.5),(0.2,0.3,0.4)\rangle \\
& \mathcal{B}=\langle u,(0.5,0.7,0.6),(0.23,0.3,0.4),(0.1,0.3,0.2)\rangle \\
& d(\mathcal{A}, \mathcal{B})=\frac{1}{3} \sum_{i=1}^{P} \omega_{i}\left[\left|\mu_{\mathcal{A}}^{i}\left(u_{i}\right)-\mu_{\mathcal{B}}^{i}\left(u_{i}\right)\right|+\left|v_{\mathcal{A}}^{i}\left(u_{i}\right)-v_{\mathcal{B}}^{i}\left(u_{i}\right)\right|+\left|w_{\mathcal{A}}^{i}\left(u_{i}\right)-w_{\mathcal{B}}^{i}\left(u_{i}\right)\right|\right] \\
& =\frac{1}{3}\left[\begin{array}{c}
\omega_{1}\left(\left|\mu_{\mathcal{A}}^{1}\left(u_{1}\right)-\mu_{\mathcal{B}}^{1}\left(u_{1}\right)\right|+\left|v_{\mathcal{A}}^{1}\left(u_{1}\right)-v_{\mathcal{B}}^{1}\left(u_{1}\right)\right|+\left|w_{\mathcal{A}}^{1}\left(u_{1}\right)-w_{\mathcal{B}}^{1}\left(u_{1}\right)\right|\right) \\
+\omega_{2}\left(\left|\mu_{\mathcal{A}}^{2}\left(u_{2}\right)-\mu_{\mathcal{B}}^{2}\left(u_{2}\right)\right|+\left|v_{\mathcal{A}}^{2}\left(u_{2}\right)-v_{\mathcal{B}}^{2}\left(u_{2}\right)\right|+\left|w_{\mathcal{A}}^{2}\left(u_{2}\right)-w_{\mathcal{B}}^{2}\left(u_{2}\right)\right|\right) \\
+\omega_{3}\left(\left|\mu_{\mathcal{A}}^{3}\left(u_{3}\right)-\mu_{\mathcal{B}}^{3}\left(u_{3}\right)\right|+\left|v_{\mathcal{A}}^{3}\left(u_{3}\right)-v_{\mathcal{B}}^{3}\left(u_{3}\right)\right|+\left|w_{\mathcal{A}}^{3}\left(u_{3}\right)-w_{\mathcal{B}}^{3}\left(u_{3}\right)\right|\right)
\end{array}\right] \\
& =\frac{1}{3}[0.2(|0.8-0.5|+|0.3-0.2|+|0.2-0.1|) \\
& +0.4(|0.5-0.7|+|0.1-0.3|+|0.3-0.3|) \\
& +0.4(|0.6-0.6|+|0.5-0.4|+|0.4-0.2|)] \\
& =\frac{1}{3}[(0.2(0.3+0.1+0.1))+(0.4(0.2+0.2))+(0.4(0.1+0.2))] \\
& d(\mathcal{A}, \mathcal{B})=0,127 \\
& d_{2}(\mathcal{A}, \mathcal{B})=\left\{\frac{1}{3} \sum_{i=1}^{P} \omega_{i}\left[\left|\mu_{\mathcal{A}}^{i}\left(u_{i}\right)-\mu_{\mathcal{B}}^{i}\left(u_{i}\right)\right|^{2}+\left|v_{\mathcal{A}}^{i}\left(u_{i}\right)-v_{\mathcal{B}}^{i}\left(u_{i}\right)\right|^{2}+\left|w_{\mathcal{A}}^{i}\left(u_{i}\right)-w_{\mathcal{B}}^{i}\left(u_{i}\right)\right|^{2}\right]\right\}^{1 / 2} \\
& =\left\{\begin{array}{l}
\frac{1}{3}\left[\omega_{1}\left(\left|\mu_{\mathcal{A}}^{1}\left(u_{1}\right)-\mu_{\mathcal{B}}^{1}\left(u_{1}\right)\right|^{2}+\left|v_{\mathcal{A}}^{1}\left(u_{1}\right)-v_{\mathcal{B}}^{1}\left(u_{1}\right)\right|^{2}+\left|w_{\mathcal{A}}^{1}\left(u_{1}\right)-w_{\mathcal{B}}^{1}\left(u_{1}\right)\right|^{2}\right)\right. \\
+\omega_{2}\left(\left|\mu_{\mathcal{A}}^{2}\left(u_{2}\right)-\mu_{\mathcal{B}}^{2}\left(u_{2}\right)\right|^{2}+\left|v_{\mathcal{A}}^{2}\left(u_{2}\right)-v_{\mathcal{B}}^{2}\left(u_{2}\right)\right|^{2}+\left|w_{\mathcal{A}}^{2}\left(u_{2}\right)-w_{\mathcal{B}}^{2}\left(u_{2}\right)\right|^{2}\right) \\
\left.+\omega_{3}\left(\left|\mu_{\mathcal{A}}^{3}\left(u_{3}\right)-\mu_{\mathcal{B}}^{3}\left(u_{3}\right)\right|^{2}+\left|v_{\mathcal{A}}^{3}\left(u_{3}\right)-v_{\mathcal{B}}^{3}\left(u_{3}\right)\right|^{2}+\left|w_{\mathcal{A}}^{3}\left(u_{3}\right)-w_{\mathcal{B}}^{3}\left(u_{3}\right)\right|^{2}\right)\right]
\end{array}\right\}^{1 / 2} \\
& =\left\{\begin{array}{l}
\frac{1}{3}\left[0.2\left(|0.8-0.5|^{2}+|0.3-0.2|^{2}+|0.2-0.1|^{2}\right)\right. \\
+0.4\left(|0.5-0.7|^{2}+|0.1-0.3|^{2}+|0.3-0.3|^{2}\right) \\
\left.+0.4\left(|0.6-0.6|^{2}+|0.5-0.4|^{2}+|0.4-0.2|^{2}\right)\right]
\end{array}\right\}^{1 / 2} \\
& =\left[\frac{1}{3}\{(0,2(0,09+0,01+0,01))+(0,4(0,04+0,04))+(0,4(0,01+0,04))\}\right]^{1 / 2} \\
& d_{2}(\mathcal{A}, \mathcal{B})=0,157
\end{aligned}
$$


Definition 9 Let

$$
\begin{aligned}
& \mathcal{A}=\{ \\
& \prec u\left(\mu_{\mathcal{A}}^{1}(u), \mu_{\mathcal{A}}^{2}(u), \ldots \mu_{\mathcal{A}}^{p}(u)\right)\left(v_{\mathcal{A}}^{1}(u), v_{\mathcal{A}}^{2}(u), \ldots v_{\mathcal{A}}^{p}(u)\right)\left(w_{\mathcal{A}}^{1}(u), w_{\mathcal{A}}^{2}(u), \ldots w_{\mathcal{A}}^{p}(u)\right) \\
& \succ: u \in \mathcal{U}\}
\end{aligned}
$$

and

$$
\begin{aligned}
& \mathcal{B}=\{ \\
& \prec u\left(\mu_{\mathcal{B}}^{1}(u), \mu_{\mathcal{B}}^{2}(u), \ldots \mu_{\mathcal{B}}^{p}(u)\right)\left(v_{\mathcal{B}}^{1}(u), v_{\mathcal{B}}^{2}(u), \ldots v_{\mathcal{B}}^{p}(u)\right)\left(w_{\mathcal{B}}^{1}(u), w_{\mathcal{B}}^{2}(u), \ldots w_{\mathcal{B}}^{p}(u)\right) \\
& \succ: u \in \mathcal{U}\}
\end{aligned}
$$

be two refined neutrosophic sets. Then, hybrid similarity measure between refined neutrosophic sets $\mathcal{A}$ and $\mathcal{B}$ denoted

$$
\begin{array}{r}
\operatorname{Hybd}(\mathcal{A}, \mathcal{B})=\varphi\left(\frac{1}{3} \sum_{i=1}^{P} \omega_{i}\left[\left|\mu_{\mathcal{A}}^{i}\left(u_{i}\right)-\mu_{\mathcal{B}}^{i}\left(u_{i}\right)\right|+\left|v_{\mathcal{A}}^{i}\left(u_{i}\right)+v_{\mathcal{B}}^{i}\left(u_{i}\right)\right|+\left|w_{\mathcal{A}}^{i}\left(u_{i}\right)-w_{\mathcal{B}}^{i}\left(u_{i}\right)\right|\right]\right) \\
+(1-\varphi)\left(\frac{1}{3} \sum_{i=1}^{P} \omega_{i}\left[\left|\mu_{\mathcal{A}}^{i}\left(u_{i}\right)-\mu_{\mathcal{B}}^{i}\left(u_{i}\right)\right|^{2}+\left|v_{\mathcal{A}}^{i}\left(u_{i}\right)+v_{\mathcal{B}}^{i}\left(u_{i}\right)\right|^{2}+\left|w_{\mathcal{A}}^{i}\left(u_{i}\right)-w_{\mathcal{B}}^{i}\left(u_{i}\right)\right|^{2}\right]\right)^{1 / 2}
\end{array}
$$

for $i=1,2, \ldots, P$. Note that similarity and distance (dissimilarity) measures are complementary: when the first increases, the second decreases. Normalized distance measure and similarity measure below are dual concepts. Thus,

$$
\delta(\mathcal{A}, \mathcal{B})=1-H y b d(\mathcal{A}, \mathcal{B})
$$

and vice versa. The properties of distance measures below are complementary to those of similarity measures.

Remark: Then, to compare hybrid similarity measures, the positive ideal refined neutrosophic solution and negative ideal refined neutrosophic solution are defined as;

$\varphi_{i}^{+}=\left\langle\frac{\max \left(\mu_{\mathcal{A}}^{1}(u), \mu_{\mathcal{A}}^{2}(u), \ldots \mu_{\mathcal{A}}^{p}(u)\right)+\min \left(v_{\mathcal{A}}^{1}(u), v_{\mathcal{A}}^{2}(u), \ldots v_{\mathcal{A}}^{p}(u)\right)+\min \left(w_{\mathcal{A}}^{1}(u), w_{\mathcal{A}}^{2}(u), \ldots w_{\mathcal{A}}^{p}(u)\right)}{3}\right\rangle$

$\varphi_{i}^{-}=\left\langle\frac{\min \left(\mu_{\mathcal{A}}^{1}(u), \mu_{\mathcal{A}}^{2}(u), \ldots \mu_{\mathcal{A}}^{p}(u)\right)+\max \left(v_{\mathcal{A}}^{1}(u), v_{\mathcal{A}}^{2}(u), \ldots v_{\mathcal{A}}^{p}(u)\right)+\max \left(w_{\mathcal{A}}^{1}(u), w_{\mathcal{A}}^{2}(u), \ldots w_{\mathcal{A}}^{p}(u)\right)}{3}\right\rangle$

respectively, for $i=1,2, \ldots, n$.

Proposition 10 The similarity measure $\delta_{p}(\mathcal{A}, \mathcal{B})$ for $p>0$ satisfies the following properties;

(HD1) $0 \leq \delta_{p}(\mathcal{A}, \mathcal{B}) \leq 1$

(HD2) $\delta_{p}(\mathcal{A}, \mathcal{B})=1$ if and only if $\mathcal{A}=\mathcal{B}$

(HD3) $\delta_{p}(\mathcal{A}, \mathcal{B})=\delta_{p}(\mathcal{B}, \mathcal{A})$;

(HD4) If $\subseteq \mathcal{B} \subseteq \mathcal{C}, \mathcal{C}$ is a refined neutrosophic in $\mathcal{U}$, then $\delta_{p}(\mathcal{A}, \mathcal{C}) \leq \delta_{p}(\mathcal{A}, \mathcal{B})$ and

$$
\delta_{p}(\mathcal{A}, \mathcal{C}) \leq \delta_{p}(\mathcal{B}, \mathcal{C})
$$


Assume that there are two refined neutrosophic

$$
\begin{aligned}
& \mathcal{A}=\{ \\
& \prec u\left(\mu_{\mathcal{A}}^{1}(u), \mu_{\mathcal{A}}^{2}(u), \ldots \mu_{\mathcal{A}}^{p}(u)\right)\left(v_{\mathcal{A}}^{1}(u), v_{\mathcal{A}}^{2}(u), \ldots v_{\mathcal{A}}^{p}(u)\right)\left(w_{\mathcal{A}}^{1}(u), w_{\mathcal{A}}^{2}(u), \ldots w_{\mathcal{A}}^{p}(u)\right) \\
& \succ: u \in \mathcal{U}\}
\end{aligned}
$$

and

$$
\begin{aligned}
& \mathcal{B}=\{ \\
& \prec u\left(\mu_{\mathcal{B}}^{1}(u), \mu_{\mathcal{B}}^{2}(u), \ldots \mu_{\mathcal{B}}^{p}(u)\right)\left(v_{\mathcal{B}}^{1}(u), v_{\mathcal{B}}^{2}(u), \ldots v_{\mathcal{B}}^{p}(u)\right)\left(w_{\mathcal{B}}^{1}(u), w_{\mathcal{B}}^{2}(u), \ldots w_{\mathcal{B}}^{p}(u)\right) \\
& \succ: u \in \mathcal{U}\}
\end{aligned}
$$

in a universe of distance $\forall u_{i} \in \mathcal{U}$. Thus, according to the relationship between the distance and the similarity measure, we can obtain the following refined neutrosophic similarity measure:

$$
\begin{aligned}
& \delta(\mathcal{A}, \mathcal{B})=1-\operatorname{Hybd}(\mathcal{A}, \mathcal{B}) \\
& =1-\left(\varphi\left(\frac{1}{3} \sum_{i=1}^{P} \omega_{i}\left[\left|\mu_{\mathcal{A}}^{i}\left(u_{i}\right)-\mu_{\mathcal{B}}^{i}\left(u_{i}\right)\right|+\left|v_{\mathcal{A}}^{i}\left(u_{i}\right)+v_{\mathcal{B}}^{i}\left(u_{i}\right)\right|+\left|w_{\mathcal{A}}^{i}\left(u_{i}\right)-w_{\mathcal{B}}^{i}\left(u_{i}\right)\right|\right]\right)+\right. \\
& \left.(1-\varphi)\left(\frac{1}{3} \sum_{i=1}^{P} \omega_{i}\left[\left|\mu_{\mathcal{A}}^{i}\left(u_{i}\right)-\mu_{\mathcal{B}}^{i}\left(u_{i}\right)\right|^{2}+\left|v_{\mathcal{A}}^{i}\left(u_{i}\right)+v_{\mathcal{B}}^{i}\left(u_{i}\right)\right|^{2}+\left|w_{\mathcal{A}}^{i}\left(u_{i}\right)-w_{\mathcal{B}}^{i}\left(u_{i}\right)\right|^{2}\right]\right)^{1 / 2}\right)
\end{aligned}
$$

Obviously, we can easily prove that $\delta_{1}(A, B)$ satisfied the properties (HD1) - (HD4) in proposition 10 by the relationship between the distance and the similarity measure and the proof of proposition 7 , which is omitted here.

Furthermore, we can also propose another refined neutrosophic similarity measure:

$$
\begin{aligned}
\delta_{2}(\mathcal{A}, \mathcal{B})=\frac{1-\operatorname{Hybd}(\mathcal{A}, \mathcal{B})}{1+\operatorname{Hybd}(\mathcal{A}, \mathcal{B})} & 1-\left(\begin{array}{l}
\varphi\left(\frac{1}{3} \sum_{i=1}^{P} \omega_{i}\left[\left|\mu_{\mathcal{A}}^{i}\left(u_{i}\right)-\mu_{\mathcal{B}}^{i}\left(u_{i}\right)\right|+\left|v_{\mathcal{A}}^{i}\left(u_{i}\right)+v_{\mathcal{B}}^{i}\left(u_{i}\right)\right|+\left|w_{\mathcal{A}}^{i}\left(u_{i}\right)-w_{\mathcal{B}}^{i}\left(u_{i}\right)\right|\right]\right)+ \\
(1-\varphi)\left(\frac{1}{3} \sum_{i=1}^{P} \omega_{i}\left[\left|\mu_{\mathcal{A}}^{i}\left(u_{i}\right)-\mu_{\mathcal{B}}^{i}\left(u_{i}\right)\right|^{2}+\left|v_{\mathcal{A}}^{i}\left(u_{i}\right)+v_{\mathcal{B}}^{i}\left(u_{i}\right)\right|^{2}+\left|w_{\mathcal{A}}^{i}\left(u_{i}\right)-w_{\mathcal{B}}^{i}\left(u_{i}\right)\right|^{2}\right]\right)^{1 / 2}
\end{array}\right) \\
= & \frac{1+\left(\begin{array}{l}
\varphi\left(\frac{1}{3} \sum_{i=1}^{P} \omega_{i}\left[\left|\mu_{\mathcal{A}}^{i}\left(u_{i}\right)-\mu_{\mathcal{B}}^{i}\left(u_{i}\right)\right|+\left|v_{\mathcal{A}}^{i}\left(u_{i}\right)+v_{\mathcal{B}}^{i}\left(u_{i}\right)\right|+\left|w_{\mathcal{A}}^{i}\left(u_{i}\right)-w_{\mathcal{B}}^{i}\left(u_{i}\right)\right|\right]\right)+ \\
(1-\varphi)\left(\frac{1}{3} \sum_{i=1}^{P} \omega_{i}\left[\left|\mu_{\mathcal{A}}^{i}\left(u_{i}\right)-\mu_{\mathcal{B}}^{i}\left(u_{i}\right)\right|^{2}+\left|v_{\mathcal{A}}^{i}\left(u_{i}\right)+v_{\mathcal{B}}^{i}\left(u_{i}\right)\right|^{2}+\left|w_{\mathcal{A}}^{i}\left(u_{i}\right)-w_{\mathcal{B}}^{i}\left(u_{i}\right)\right|^{2}\right]\right)^{1 / 2}
\end{array}\right)}{}
\end{aligned}
$$

Then, the similarity measure $\delta_{2}(\mathcal{A}, \mathcal{B})$ also satisfied the properties (HD1) - (HD4) in Proposition 2 .

Proof: It is easy to see that $\delta_{2}(\mathcal{A}, \mathcal{B})$ satisfies the properties(HD1) - (HD3). Therefore, we only property have to prove (HD4)

As we obtain $\delta_{p}(\mathcal{A}, \mathcal{B}) \leq \delta_{p}(\mathcal{A}, \mathcal{C})$ and $\delta_{p}(\mathcal{B}, \mathcal{C}) \leq \delta_{p}(\mathcal{A}, \mathcal{C})$ for $p>0$ from the property (H4) in proposition 1 , there are $1-\delta_{p}(\mathcal{A}, \mathcal{B}) \geq 1-\delta_{p}(\mathcal{A}, \mathcal{C}), 1-\delta_{p}(\mathcal{B}, \mathcal{C}) \geq 1-\delta_{p}(\mathcal{A}, \mathcal{C})$.

$1+\delta_{p}(\mathcal{A}, \mathcal{B}) \leq 1+\delta_{p}(\mathcal{A}, \mathcal{C})$ and $1+\delta_{p}(\mathcal{B}, \mathcal{C}) \leq 1+\delta_{p}(\mathcal{A}, \mathcal{C})$. Then, there are the following inequalities:

$$
\frac{1-d_{p}(\mathcal{A}, \mathcal{B})}{1+d_{p}(\mathcal{A}, \mathcal{B})} \geq \frac{1-d_{p}(\mathcal{A}, \mathcal{C})}{1+d_{p}(\mathcal{A}, \mathcal{C})} \quad \text { and } \quad \frac{1-d_{p}(\mathcal{B}, \mathcal{C})}{1+d_{p}(\mathcal{B}, \mathcal{C})} \geq \frac{1-d_{p}(\mathcal{A}, \mathcal{C})}{1+d_{p}(\mathcal{A}, \mathcal{C})}
$$


Then, there are $\delta_{p}(\mathcal{A}, \mathcal{C}) \leq \delta_{p}(\mathcal{A}, \mathcal{B})$ and $\delta_{p}(\mathcal{A}, \mathcal{C}) \leq \delta_{p}(\mathcal{B}, \mathcal{C})$. Hence, the property (HD4) is satisfied.

Example 11: Assume that we have the following two refined neutrosophic hybrid similarity measure in a universe of discourse $u \in \mathcal{U}$; let $\omega_{1}=0.2, \omega_{2}=0.3, \omega_{3}=0.5$

$$
\begin{aligned}
& \mathcal{A}=\langle u,(0.5,0.5,0.3),(0.8,0.1,0.2),(0.2,0.8,0.9)\rangle \\
& \mathcal{B}=\langle u,(0.5,0.1,0.6),(0.2,0.3,0.9),(0.6,0.3,0.2)\rangle \\
& \operatorname{Hybd}(\mathcal{A}, \mathcal{B})=\left(\varphi\left(\frac{1}{3} \sum_{i=1}^{P} \omega_{i}\left[\left|\mu_{\mathcal{A}}^{i}\left(u_{i}\right)-\mu_{\mathcal{B}}^{i}\left(u_{i}\right)\right|+\left|v_{\mathcal{A}}^{i}\left(u_{i}\right)+v_{\mathcal{B}}^{i}\left(u_{i}\right)\right|+\left|w_{\mathcal{A}}^{i}\left(u_{i}\right)-w_{\mathcal{B}}^{i}\left(u_{i}\right)\right|\right]\right)+\right. \\
& \left.(1-\varphi)\left(\frac{1}{3} \sum_{i=1}^{P} \omega_{i}\left[\left|\mu_{\mathcal{A}}^{i}\left(u_{i}\right)-\mu_{\mathcal{B}}^{i}\left(u_{i}\right)\right|^{2}+\left|v_{\mathcal{A}}^{i}\left(u_{i}\right)+v_{\mathcal{B}}^{i}\left(u_{i}\right)\right|^{2}+\left|w_{\mathcal{A}}^{i}\left(u_{i}\right)-w_{\mathcal{B}}^{i}\left(u_{i}\right)\right|^{2}\right]\right)^{1 / 2}\right) \\
& =\left(\begin{array}{c}
\varphi\left(\frac{1}{3}\left[\begin{array}{c}
\omega_{1}\left(\left|\mu_{\mathcal{A}}^{1}\left(u_{1}\right)-\mu_{\mathcal{B}}^{1}\left(u_{1}\right)\right|+\left|v_{\mathcal{A}}^{1}\left(u_{1}\right)-v_{\mathcal{B}}^{1}\left(u_{1}\right)\right|+\left|w_{\mathcal{A}}^{1}\left(u_{1}\right)-w_{\mathcal{B}}^{1}\left(u_{1}\right)\right|\right) \\
+\omega_{2}\left(\left|\mu_{\mathcal{A}}^{2}\left(u_{2}\right)-\mu_{\mathcal{B}}^{2}\left(u_{2}\right)\right|+\left|v_{\mathcal{A}}^{2}\left(u_{2}\right)-v_{\mathcal{B}}^{2}\left(u_{2}\right)\right|+\left|w_{\mathcal{A}}^{2}\left(u_{2}\right)-w_{\mathcal{B}}^{2}\left(u_{2}\right)\right|\right) \\
+\omega_{3}\left(\mid \begin{array}{l}
\mu_{\mathcal{A}}^{3}\left(u_{3}\right)-\mu_{\mathcal{B}}^{3}\left(u_{3}\right)|+| v_{\mathcal{A}}^{3}\left(u_{3}\right)-v_{\mathcal{B}}^{3}\left(u_{3}\right)|+| w_{\mathcal{A}}^{3}\left(u_{3}\right)-w_{\mathcal{B}}^{3}\left(u_{3}\right)
\end{array}\right)
\end{array}\right]\right) \\
+(1-\varphi)\left\{\begin{array}{c}
\frac{1}{3}\left[\omega_{1}\left(\left|\mu_{\mathcal{A}}^{1}\left(u_{1}\right)-\mu_{\mathcal{B}}^{1}\left(u_{1}\right)\right|^{2}+\left|v_{\mathcal{A}}^{1}\left(u_{1}\right)-v_{\mathcal{B}}^{1}\left(u_{1}\right)\right|^{2}+\left|w_{\mathcal{A}}^{1}\left(u_{1}\right)-w_{\mathcal{B}}^{1}\left(u_{1}\right)\right|^{2}\right)\right. \\
+\omega_{2}\left(\left|\mu_{\mathcal{A}}^{2}\left(u_{2}\right)-\mu_{\mathcal{B}}^{2}\left(u_{2}\right)\right|^{2}+\left|v_{\mathcal{A}}^{2}\left(u_{2}\right)-v_{\mathcal{B}}^{2}\left(u_{2}\right)\right|^{2}+\left|w_{\mathcal{A}}^{2}\left(u_{2}\right)-w_{\mathcal{B}}^{2}\left(u_{2}\right)\right|^{2}\right) \\
\left.+\omega_{3}\left(\left|\mu_{\mathcal{A}}^{3}\left(u_{3}\right)-\mu_{B}^{3}\left(u_{3}\right)\right|^{2}+\left|v_{\mathcal{A}}^{3}\left(u_{3}\right)-v_{\mathcal{B}}^{3}\left(u_{3}\right)\right|^{2}+\left|w_{\mathcal{A}}^{3}\left(u_{3}\right)-w_{\mathcal{B}}^{3}\left(u_{3}\right)\right|^{2}\right)\right]
\end{array}\right\}
\end{array}\right\} \\
& =\left(\begin{array}{c}
0.3\left(\frac{1}{3}\left[\begin{array}{l}
0.2(|0.5-0.5|+|0.8-0.2|+|0.2-0.6|) \\
+0.3(|0.5-0.1|+|0.1-0.3|+|0.8-0.3|) \\
+0.5(|0.3-0.6|+|0.2-0.9|+|0.9-0.2|)
\end{array}\right]\right) \\
+0.7\left\{\begin{array}{c}
\frac{1}{3}\left[0.2\left(|0.5-0.5|^{2}+|0.8-0.2|^{2}+|0.2-0.6|^{2}\right)\right. \\
+0.3\left(|0.5-0.1|^{2}+|0.1-0.3|^{2}+|0.8-0.3|^{2}\right) \\
\left.+0.5\left(|0.3-0.6|^{2}+|0.2-0.9|^{2}+|0.9-0.2|^{2}\right)\right]
\end{array}\right\}
\end{array}\right) \\
& =0.3\left(\frac{1}{3}[(0,2(0,6+0,4))+(0,3(0,4+0,2+0,5))+(0,5(0,3+0,7+0,7))]\right) \\
& +0.7\left(\left[\frac{1}{3}\{(0,2(0,36+0,16))+(0,3(0,16+0,04+0,25))+(0,5(0,09+0,49+0,49))\}\right]^{1 / 2}\right)
\end{aligned}
$$

$\operatorname{Hybd}(\mathcal{A}, \mathcal{B})=0.4935$

$\delta(\mathcal{A}, \mathcal{B})=1-\operatorname{Hybd}(\mathcal{A}, \mathcal{B})$

$\delta(\mathcal{A}, \mathcal{B})=0.5065$

\section{Medical Diagnosis Using the Hybrid Similarity Measure}

We consider a medical diagnosis problem from practical point of view for illustration of the proposed approach. Medical diagnosis comprises of uncertainties and increased volume of information available to physicians from new medical technologies. The process of classifying different set of symptoms under a single name of a disease is a very difficult task. In some practical situations, there exists possibility of each element within a lower and an upper approximation of refined neutrosophic sets. It can deal with the medical diagnosis involving more indeterminacy. Actually this approach is more flexible and easy to use. The proposed similarity measure among the patients versus symptoms and symptoms versus diseases will provide the proper medical diagnosis. The main feature of this proposed approach is that it considers truth membership, indeterminate and false membership of each element between two approximations of refined neutrosophic sets by taking one time inspection for diagnosis. 
Now, an example of a medical diagnosis is presented. Let $P=\{$ Erol, Harun, Deniz $\}$ be a set of patients, $D=\{$ Viral Fever, Tuberculosis, Throat $\}$ disease be a set of diseases and $\mathrm{S}=\{$ Throat pain, headache, body pain $\}$ be a set of symptoms. Our solution is to examine the patient at different time intervals (three times a day), which in turn give arise to different truth membership, indeterminate and false membership function for each patient in Table 1-Table 8. Let $\omega_{1}=0.2, \omega_{2}=0.5, \omega_{3}=0.3$. Let the samples be taken at three different timings in a day (in 07:00, 15:00 and 23:00)

Table 1: Q (The Relation Between Patient and Symptoms)

\begin{tabular}{|c|c|c|c|}
\hline $\mathrm{Q}$ & Throat pain & Headache & Body Pain \\
\hline \multirow{3}{*}{ Erol } & $\langle(0.1,0.3,0.6),(0.3,0.8,0.3)(0.3,0.3,0.4)\rangle$ & $\langle(0.1,0.5,0.6),(0.3,0.5,0.8)(0.9,0.3,0.7)\rangle$ & $\langle(0.7,0.2,0.1),(0.5,0.6,0.3)(0.2,0.3,0.4)\rangle$ \\
& $\langle(0.8,0.2,0.9),(0.7,0.4,0.4)(0.2,0.7,0.5)\rangle$ & $\langle(0.5,0.5,0.5),(0.6,0.3,0.5)(0.4,0.3,0.5)\rangle$ & $\langle(0.5,0.5,0.3),(0.3,0.4,0.5)(0.3,0.2,0.2)\rangle$ \\
& $\langle(0.9,0.5,0.6),(0.3,0.3,0.5)(0.2,0.3,0.4)\rangle$ & $\langle(0.6,0.7,0.8),(0.5,0.3,0.5)(0.5,0.4,0.1)\rangle$ & $\langle(0.1,0.3,0.1),(0.6,0.9,0.3)(0.2,0.4,0.4)\rangle$ \\
\hline \multirow{3}{*}{ Harun } & $\langle(0.6,0.5,0.5),(0.5,0.4,0.5)(0.4,0.3,0.4)\rangle$ & $\langle(0.1,0.5,0.6),(0.6,0.7,0.5)(0.3,0.3,0.4)\rangle$ & $\langle(0.8,0.6,0.6),(0.5,0.6,0.4)(0.4,0.3,0.5)\rangle$ \\
& $\langle(0.3,0.5,0.6),(0.6,0.9,0.4)(0.5,0.3,0.9)\rangle$ & $\langle(0.3,0.3,0.6),(0.6,0.7,0.5)(0.3,0.3,0.9)\rangle$ & $\langle(0.5,0.2,0.3),(0.9,0.6,0.5)(0.9,0.3,0.4)\rangle$ \\
& $\langle(0.8,0.7,0.1),(0.8,0.7,0.5)(0.3,0.7,0.4)\rangle$ & $\langle(0.8,0.5,0.6),(0.5,0.8,0.5)(0.8,0.5,0.4)\rangle$ & $\langle(0.8,0.5,0.7),(0.6,0.5,0.4)(0.5,0.3,0.4)\rangle$ \\
\hline \multirow{3}{*}{ Deniz } & $\langle(0.8,0.6,0.6),(0.6,0.6,0.6)(0.6,0.7,0.5)\rangle$ & $\langle(0.1,0.5,0.6),(0.9,0.6,0.8)(0.9,0.5,0.7)\rangle$ & $\langle(0.4,0.5,0.6),(0.7,0.6,0.6)(0.7,0.8,0.6)\rangle$ \\
& $\langle(0.1,0.3,0.6),(0.8,0.8,0.7)(0.8,0.5,0.9)\rangle$ & $\langle(0.7,0.4,0.2),(0.9,0.8,0.8)(0.6,0.8,0.5)\rangle$ & $\langle(0.5,0.2,0.3),(0.8,0.9,0.7)(0.9,0.5,0.5)\rangle$ \\
& $\langle(0.8,0.5,0.6),(0.6,0.7,0.9)(0.6,0.9,0.5)\rangle$ & $\langle(0.9,0.5,0.7),(0.6,0.8,0.7)(0.5,0.8,0.6)\rangle$ & $\langle(0.7,0.2,0.1),(0.8,0.6,0.9)(0.5,0.9,0.9)\rangle$ \\
\hline
\end{tabular}

Table 2: R (The Relation Among Symptoms and Diseases)

\begin{tabular}{|l|c|c|c|}
\hline \multicolumn{1}{|c|}{ R } & Viral Fever & Tuberculosis & Typhoid \\
\hline Throat pain & $\langle(0.2,0.5,0.6),(0.3,0.1,0.6)(0.2,0.8,0.4)\rangle$ & $\langle(0.6,0.5,0.7),(0.2,0.1,0.5)(0.4,0.3,0.4)\rangle$ & $\langle(0.1,0.5,0.6),(0.3,0.1,0.2)(0.5,0.3,0.7)\rangle$ \\
\hline Headache & $\langle(0.4,0.5,0.6),(0.2,0.7,0.5)(0.7,0.4,0.1)\rangle$ & $\langle(0.7,0.2,0.1),(0.5,0.7,0.3)(0.3,0.2,0.4)\rangle$ & $\langle(0.3,0.2,0.3),(0.3,0.1,0.8)(0.9,0.3,0.4)\rangle$ \\
\hline Body Pain & $\langle(0.9,0.5,0.7),(0.6,0.9,0.1)(0.5,0.9,0.4)\rangle$ & $\langle(0.7,0.5,0.6),(0.3,0.1,0.5)(0.5,0.3,0.9)\rangle$ & $\langle(0.8,0.5,0.6),(0.3,0.1,0.5)(0.2,0.3,0.4)\rangle$ \\
\hline
\end{tabular}

Table 3: The Hamming Weighted Distance Refined Neutrosophic Sets $Q$ and $R$

\begin{tabular}{|c|c|c|c|}
\hline Hamming weighted & Viral Fever & Tuberculosis & Typhoid \\
\hline Erol & 0,231111 & $\mathbf{0 , 2 2 2 2 2 2}$ & 0,323333 \\
\hline Harun & $\mathbf{0 , 1 7 4 4 4 4}$ & 0,222222 & 0,342222 \\
\hline Deniz & 0,316667 & $\mathbf{0 , 2 1 1 1 1 1}$ & 0,364444 \\
\hline Optimal-Erol(Tuberculosis);Harun(Viral Fever); Deniz(Tuberculosis) \\
\hline
\end{tabular}

Table 4: The Weighted Euclidean Distance Refined Neutrosophic Sets Q and R

\begin{tabular}{|c|c|c|c|}
\hline Weighted Euclidean distance & Viral Fever & Tuberculosis & Typhoid \\
\hline Erol & $\mathbf{0 , 0 1 0 9 5 7}$ & 0,01599 & 0,027775 \\
\hline Harun & $\mathbf{0 , 0 0 3 1 3 1}$ & 0,006728 & 0,027159 \\
\hline Deniz & 0,023368 & $\mathbf{0 , 0 0 8 0 5 4}$ & 0,032489 \\
\hline \multicolumn{2}{|c|}{ Optimal-Erol(Viral Fever);Harun( Viral Fever); Deniz( Tuberculosis) } \\
\hline
\end{tabular}


Table 5: The Weighted Hybrid Distance Refined Neutrosophic Sets $Q$ and $R$ with $\varphi_{1}^{+}=0.43$

\begin{tabular}{|c|c|c|c|}
\hline Weighted hybrid & Viral Fever & Tuberculosis & Typhoid \\
\hline Erol & $\mathbf{0 , 1 0 5 6 2 3}$ & 0,133542 & 0,196243 \\
\hline Harun & $\mathbf{0 , 1 0 0 7 8}$ & 0,12956 & 0,206745 \\
\hline Deniz & 0,190548 & $\mathbf{0 , 1 2 3 7 9 7}$ & 0,221704 \\
\hline \multicolumn{2}{|c|}{ Optimal-Erol(Viral Fever);Harun( Viral Fever); Deniz( Tuberculosis) } \\
\hline
\end{tabular}

Table 6: The Weighted Similarity Measure Hybrid Distance Refined Neutrosophic Sets $Q$ and $R$ with $\varphi_{1}^{+}=0.43$.

\begin{tabular}{|c|c|c|c|}
\hline Weighted similarity Measure hybrid & Viral Fever & Tuberculosis & Typhoid \\
\hline Erol & $\mathbf{0 , 8 9 4 3 7 7}$ & 0,866458 & 0,803757 \\
\hline Harun & $\mathbf{0 , 8 9 9 2 2}$ & 0,87044 & 0,793255 \\
\hline Deniz & 0,809452 & $\mathbf{0 , 8 7 6 2 0 3}$ & 0,778296 \\
\hline \multicolumn{2}{|c|}{ Optimal-Erol(Viral Fever); Harun( Viral Fever); Deniz( Tuberculosis) } \\
\hline
\end{tabular}

Table 7: The Weighted Similarity Measure Hybrid Distance Refined Neutrosophic Sets $Q$ and $R$ with $\varphi_{2}^{+}=0.5$

\begin{tabular}{|c|c|c|c|}
\hline Weighted similarity Measure hybrid & Viral Fever & Tuberculosis & Typhoid \\
\hline Erol & 0,878966 & $\mathbf{0 , 8 8 0 8 9 4}$ & 0,824446 \\
\hline Harun & $\mathbf{0 , 9 1 1 2 1 2}$ & 0,885525 & 0,815309 \\
\hline Deniz & 0,829983 & $\mathbf{0 , 8 9 0 4 1 7}$ & 0,801533 \\
\hline \multicolumn{2}{|c|}{ Optimal-Erol(Tuberculosis);Harun( Viral Fever); Deniz( Tuberculosis) } \\
\hline
\end{tabular}

Table 8: The Weighted Similarity Measure Hybrid Distance Refined Neutrosophic Sets $Q$ and $R$ with $\varphi_{2}^{+}=0.67$

\begin{tabular}{|c|c|c|c|}
\hline Weighted similarity Measure hybrid & Viral Fever & Tuberculosis & Typhoid \\
\hline Erol & 0,84154 & $\mathbf{0 , 9 1 5 9 5 3}$ & 0,874691 \\
\hline Harun & $\mathbf{0 , 9 4 0 3 3 5}$ & 0,922159 & 0,86887 \\
\hline Deniz & 0,879843 & $\mathbf{0 , 9 2 4 9 3 7}$ & 0,857965 \\
\hline \multicolumn{2}{|c|}{ Optimal-Erol(Tuberculosis); Harun( Viral Fever); Deniz( Tuberculosis) } \\
\hline
\end{tabular}

\section{Conclusion}

In this paper, a new hybrid similarity measure and a weighted hybrid similarity measure for refined neutrosophic sets are presented and some of its basic properties are discussed. The 
proposed hybrid similarity measure enriches the theories and techniques for measuring the degree of hybrid similarity between refined neutrosophic sets. This measure greatly reduces the influence of imprecise measures and provides an extremely intuitive quantification. The effectiveness of the proposed hybrid similarity measure is demonstrated in a numerical example with the help of measure of performance and measure of error (refer to Table 1-8). Moreover, medical diagnosis problems have been exhibited through a hypothetical case study by using this proposed hybrid similarity measure. The authors hope that the proposed concept can be applied in solving realistic multi-criteria decision making problems.

\section{Compliance with Ethical Standards}

The authors declare that there is no conflict of interests regarding the publication of this paper.

\section{References}

[1] Zadeh, L. A. Fuzzy Sets, Inform. and Control.1965.8: 338-353.

[2] Atanassov, K. Intuitionistic fuzzy sets. Fuzzy Sets and Systems. 1986. 20: 87-96.

[3] Smarandache, F. A. Unifying Field in Logics Neutrosophy: Neutrosophic Probability, Set and Logic. Rehoboth: American Research Press. 1998.

[4] Shinoj T. K. and S. J. John, Intuitionistic fuzzy multisets and its application in medical diagnosis, World Academy of Science, Engineering and Technology. 2012. 6(2012): 1-28.

[5] Sebastian S. and T. V. Ramakrishnan, Multi-Fuzzy Sets, International Mathematical Forum. 2010. 5(50): 2471-2476.

[6] Rajarajeswari, P. and Uma, N. Correlation measure for intuitionistic fuzzy multi sets. International Journal of Research in Engineering and Technology. 2014. 3(1), 611-617.

[7] Rajarajeswari, P. and Uma, N. On distance and similarity measures of intuitionistic fuzzy multi set. IOSR Journal of Mathematics. 2013. 5(4): 19-23.

[8] Rajarajeswari, P. and Uma, N. A study of normalized geometric and normalized hamming distance measures in intuitionistic fuzzy multi sets. International Journal of Science and Research, Engineering and Technology. 2013. 2(11): 76-80.

[9] Rajarajeswari, P. and Uma, N. Intuitionistic fuzzy multi relations. International Journal of Mathematical Archives.2013, 4(10), 244-249.

[10] Rajarajeswari P. and Uma, N. Zhang and Fu's similarity measure on intuitionistic fuzzy multi sets.International Journal of Innovative Research in Science, Engineering and Technology. 2014. 3(5): 12309-12317.

[11] Deli, I., Broumi, S. and Ali, M. Neutrosophic soft multi-set theory and its decision making. Neutrosophic Sets and Systems. 2014. 5: 65-76.

[12] Deli, I. Refined neutrosophic sets and refined neutrosophic soft sets: theory and applications. Handbook of Research on Generalized and Hybrid Set Structures and Applications for Soft Computing. 2016. 321-343.

[13] Deli,I., Broumi, V. and Smarandache, F. On neutrosophic refined sets and their applications in medical diagnosis, Journal of New Theory. 2015. 6(2015): 88-98. 
[14] Deli, I., Eraslan, S., and Çaman, N. Ivnpiv-Neutrosophic soft sets and their decision making based on similarity measure. Neural Computing and Applications. 2016: 1-17.

[15] Fua, J. and Yeb, J. Simplified neutrosophic exponential similarity measures for the initial evaluation/diagnosis of benign prostatic hyperplasia symptom. Symmetry. 2017. 9(8): 154 .

[16] Fathi, S., ElGhawalby, H., and Salama, A. A. A Neutrosophic Graph Similarity Measures. New Trends in Neutrosophic Theories and Applications.2016.1

[17] Karaaslan, F. Correlation coefficients of single-valued neutrosophic refined soft sets and their applications in clustering analysis. Neural Computing and Applications, 2016. 1-13.

[18] Smarandache, F. n-valued refined neutrosophic logic and its applications in physics. Progress in Physics. 2013. 4: 143-146.

[19] Wang H, Smarandache F. Y., Zhang, Q. and Sunderraman R. Single valued neutrosophic sets. Multispace and Multistructure. 2010. 4: 410-413

[20] Athar, K. A neutrosophic multi-criteria decision making method. New Mathematics and Natural Computation. 2014. 10(02): 143-162.

[21] Broumi S. and F. Smarandache. Several similarity measures of neutrosophic sets. Neutrosophic Sets and Systems. 2013. 1(1): 54-62.

[22] Deli, I., Broumi S. Neutrosophic soft matrices and NSM-decision making. Journal of Intelligent and Fuzzy Systems. 2015. 28: 2233-2241.

[23] Karaaslan, F. Correlation coefficient between possibility neutrosophic soft sets. Math. Sci. Lett. 2016. 5/1, 71-74.

[24] Liu, P. and Wang, Y. Multiple attribute decision-making method based on single-valued neutrosophic normalized weighted Bonferroni mean. Neural Computing and Applications. 2014. $25(7-8):$ 2001-2010.

[25] Mondal, K. and Pramanik, S. Neutrosophic tangent similarity measure and its application to multiple attribute decision making. Neutrosophic Sets and Systems. 2015. 9: 85-92.

[26] Ptracu, V. Refined neutrosophic information based on truth, falsity, ignorance, contradiction and hesitation. Neutrosophic Sets and Systems. 2016. 11:57-65.

[27] Pramanik, S. and Mondal, K. Cotangent similarity measure of rough neutrosophic sets and its application to medical diagnosis. Journal of New Theory. 2015. 4: 90-102.

[28] Pramanik, S., Biswas, P., Giri, B. C. (2015). Hybrid vector similarity measures and their applications to multi-attribute decision making under neutrosophic environment.Neural computing and Applications. 2015. 1-14.

[29] Pramanik, S. and Mondal, K. Cosine similarity measure of rough neutrosophic sets and its application in medical diagnosis. Global Journal of Advanced Research. 2015. 2(1): 212-220.

[30] Smarandache F. Neutrosophic set, a generalisation of the intuitionistic fuzzy sets. Int. J. Pure Appl Math. 2005. 24:287-297.

[31] Sahin, M., Deli, I. and Ulucay, V. Jaccard vector similarity measure of bipolar neutrosophic set based on multi-criteria decision making. International Conference on NaturalScience and Engineering (ICNASE'16). 2016. March 19-20. Kilis. 
[32] Ye, J. Vector similarity measures of simplified neutrosophic sets and their application in multicriteria decision making. International Journal of Fuzzy Systems. 2014. 16(2): 204215.

[33] Ye, J. Improved correlation coefficients of single valued neutrosophic sets and interval neutrosophic sets for multiple attribute decision making. J. Intell. Fuzzy Syst. 2014. 27: 2453-2462

[34] Ye, J. and Zhang, Q. S. Single valued neutrosophic similarity measures for multiple attribute decision making. Neutrosophic Sets and Systems. 2014. 2: 48-54.

[35] Ye, J. and Fu, J. Multi-period medical diagnosis method using a single valued neutrosophic similarity measure based on tangent function. Computer Methods and Programs in Biomedicine (2016). 123:142-149.

[36] Jun, Y. E. Single-valued neutrosophic similarity measures based on cotangent function and their application in the fault diagnosis of steam turbine. Soft Computing. 2017. 21(3): 817-825.

[37] Ye, S., Fu, J. and Ye, J. Medical diagnosis using distance-based similarity measures of single valued neutrosophic multisets. Neutrosophic Sets and Systems. 2015. 7: 47-52.

[38] Smarandache, F. Neutrosphic multiset applied in physical processes, actualization of the internet of things. FIAP Industrial Physics Conference, Monterey, California. Jan. 2017.

[39] Smarandache, F. Neutrosophic Perspectives: Triplets, Duplets, Multisets, Hybrid Operators, Modal Logic, Hedge Algebras and Applications. Pons Editions, Bruxelles, 323 p., 2017. chapter x: 115-123.

[40] Bakbak, Derya; Uluçay, Vakkas and Sahin, Memet. Neutrosophic soft expert multiset and their application to multiple criteria decision making. Mathematics. 2019. 7(1): 50.

[41] Ulucay, Vakkas, Sahin, Mehmet, and Olgun, Necati. Time-neutrosophic soft expert sets and its decision making problem. Matematika. 2018. 34(2): 246-260.

[42] Uluçay, V., Kiliç, A., Yildiz, I. and Sahin, M. A new approach for multi-attribute decisionmaking problems in bipolar neutrosophic sets. Neutrosophic Sets Syst. 2018. 23: 142-159.

[43] Sahin, M., Olgun, N., Uluçay, V., Kargın, A., and Smarandache, F. A new similarity measure based on falsity value between single valued neutrosophic sets based on the centroid points of transformed single valued neutrosophic numbers with applications to pattern recognition. Infinite Study. 2017.

[44] Broumi, S., Bakali, A., Talea, M., Smarandache, F., Singh, P. K., Uluçay, V. and Khan, M. Bipolar Complex Neutrosophic Sets and Its Application in Decision Making Problem. In Fuzzy Multi-criteria Decision-Making Using Neutrosophic Sets. Springer, Cham. 2019. 677-710).

[45] Ye, S. and Ye, J. Dice similarity measure between single valued neutrosophic multisets anf its application in medical diagnosis. Neutrosophic Sets and Systems. 2014. 6: 49-54. 